\title{
Mechanistic Insights into the Oxidation of Veratryl Alcohol with Co(salen) and Oxygen in Aqueous Media: An in-situ Spectroscopic Study
}

\author{
Kaisa Kervinen, ${ }^{[a, b]}$ Heikki Korpi, ${ }^{[a]}$ J. Gerbrand Mesu, ${ }^{[b]}$ Fouad Soulimani, ${ }^{[b]}$ Timo Repo, ${ }^{*[a]}$ \\ Bernhard Rieger, ${ }^{[c]}$ Markku Leskelä, ${ }^{[a]}$ and Bert M. Weckhuysen*[b]
}

\author{
Keywords: Cobalt complexes / Schiff bases / Homogeneous catalysis / IR spectroscopy / Raman spectroscopy / UV/Vis \\ spectroscopy
}

\begin{abstract}
Many transition-metal complexes can perform catalytic oxidations, but their corresponding reaction pathways are still not clear. In this study, the mechanism of Co(salen)-catalyzed [salen $=N, N^{\prime}$-bis(salicylidene)ethylenediamine] oxidization of veratryl alcohol (3,4-dimethoxybenzyl alcohol) by dioxygen in alkaline aqueous solution was elucidated with insitu ATR-IR, Raman and UV/Vis spectroscopy. The mechanism of this reaction seems to start by formation of a bis$\mu$-hydroxo[(Co(salen) $]_{2}$ species, which explains the dramatic effect of $\mathrm{pH}$ on the reaction rate. Substrate coordination to this species leads to formation of a cobalt-bound veratryl alkoxo intermediate, to which oxygen molecule can bind. For-
\end{abstract}

mation of a $\mu$-peroxo bridge between two such Co(salen) substrate units is observed in the UV/Vis spectra. Transfer of a hydrogen atom from the substrate to the peroxo bridge results in detachment of the product aldehyde and regeneration of the initial bis- $\mu$-hydroxo[(Co(salen) $]_{2}$ species. In the overall cycle two substrate molecules are oxidized to aldehyde and molecular oxygen is reduced to water. The ratelimiting step is the detachment of the product molecule, which is aided by the methoxy substituents in the aromatic ring of the benzylic alcohol.

() Wiley-VCH Verlag GmbH \& Co. KGaA, 69451 Weinheim, Germany, 2005)

\section{Introduction}

Activation of molecular oxygen by transition-metal complexes for selective oxidations in water is an environmentally benign and economical alternative for many present day industrial processes. ${ }^{[1-3]}$ Nevertheless, organic solvents are more commonly used, as stability and solubility of transition-metal complexes is often a problem in aqueous solutions. ${ }^{[2-7]}$ Despite the importance, the mechanisms of transition-metal complex-catalyzed oxidation reactions are still not fully understood. ${ }^{[4]}$ For example, Co(salen) [salen = $N, N^{\prime}$-bis(salicylidene)ethylenediamine] has long been known to reversibly bind and activate dioxygen and its oxygen adducts are well characterized, ${ }^{[8-10]}$ but it is not clear how these catalytic reactions proceed. ${ }^{[4,11]}$ It has been suggested that the mononuclear superoxo species is usually responsible for catalyst activity in the oxidation of phenolic substrates, but no direct evidence is yet available. ${ }^{[3,4,12-14]}$ There are less examples on the oxidation of benzylic substrates. It has been noted that the reaction mechanisms de-

[a] Laboratory of Inorganic Chemistry, Department of Chemistry, University of Helsinki,

P. O. Box 55, 00014 Helsinki, Finland

E-mail: timo.repo@helsinki.fi

[b] Department of Inorganic Chemistry and Catalysis, Debye Institute, University of Utrecht,

Sorbonnelaan 16, 3584 CA Utrecht, The Netherlands

E-mail: b.m.weckhuysen@chem.uu.nl

[c] Department of Materials and Catalysis, University of Ulm, Albert-Einstein-Allee 11, 89069 Ulm, Germany pend greatly on the nature of the substrate and its substituents. ${ }^{[3,15]}$

In this study, $\mathrm{Co}$ (salen) is used as a catalyst to oxidize a lignin model compound, veratryl alcohol (3,4-dimethoxybenzyl alcohol), to an aldehyde in alkaline water solution. Selective oxidation of lignin would be attractive for catalytic pulp bleaching ${ }^{[16-18]}$ and for the search of renewable raw materials. ${ }^{[3,12]}$ This reaction proceeds in the presence of molecular oxygen to reach TOF $=10-15$ at optimum conditions $(\mathrm{TOF}=$ turnover frequency, moles of product per mol of catalyst per hour). ${ }^{[16]}$ The effect of reaction conditions observed previously for this system suggest that a mononuclear superoxo complex is not responsible for catalytic activity. ${ }^{[16-17]}$ However, direct proof for the intermediates involved in the catalytic cycle is lacking. Temperature and $\mathrm{pH}$ have a dramatic effect on the catalytic activity of this system, and thus it is vital that the mechanism is studied under real experimental conditions. Therefore, the Co(salen)-catalyzed reaction (Scheme 1) has been studied with a combination of in-situ ATR-IR, Raman and UV/Vis spectroscopy in aqueous solution at $\mathrm{pH} 12$ and $80{ }^{\circ} \mathrm{C}$.

The use of complementary techniques is a powerful way to monitor a catalytic reaction. ${ }^{[20-21]}$ Different species show more intense absorptions in different regions of the electromagnetic spectrum. Electronic transitions usually give rise to absorption or emission in the ultraviolet or visible regions, whereas molecular vibrations give rise to absorption bands throughout most of the infrared region. Symmetrical 
<smiles>COc1ccc(CO)cc1OC</smiles>

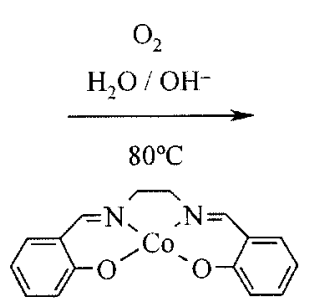

Scheme 1. Oxidation of veratryl alcohol by Co(salen) (1) and dioxygen in water.

molecules can be totally IR inactive, but exhibit intense vibrations in the Raman spectrum. ${ }^{[19]}$ In this work, UV/Vis spectroscopy was used to study the reaction at low concentrations, whereas ATR-IR and Raman techniques require higher concentrations. Based on the combination of results, a mechanism for veratryl alcohol oxidation by Co(salen) in alkaline aqueous solution is proposed (Scheme 1).

\section{Results and Discussion}

\section{ATR-IR Spectroscopy}

Carbonyl compounds have distinct vibrations in the IR range, and therefore it is attractive to study the veratraldehyde formation by in-situ IR spectroscopy. ${ }^{[22-24]}$ Metaldioxygen complexes have also been extensively characterized by vibrational spectroscopic techniques. ${ }^{[24-28]}$ [Co(salen) $\mathrm{B}_{2} \mathrm{O}_{2}(\mathrm{~B}=$ py, pyO, DMF or other axial base) exhibits $v(\mathrm{O}-\mathrm{O})$ at $888-910 \mathrm{~cm}^{-1}$ and the oxygen bridge is regarded as a peroxo bridge. $[\mathrm{Co}(\text { salen })]_{2} \mathrm{O}_{2}$ exhibits $v(\mathrm{O}-\mathrm{O})$ at $1010 \mathrm{~cm}^{-1}$ and this bridge is regarded as being an intermediate between peroxo and superoxo species. ${ }^{[27]}$ The superoxo-cobalt complexes exhibit $v(\mathrm{O}-\mathrm{O})$ at a typical range of $1075-1122 \mathrm{~cm}^{-1}$. [28]

Figure 1 shows a typical set of ATR-IR spectra obtained for the $\mathrm{Co}($ salen)-catalyzed oxidation of veratryl alcohol with oxygen in water as a function of time. Mainly two vibrations are observed to form during the reaction. As expected, the aldehyde $\mathrm{C}=\mathrm{O}$ vibration peak is appearing at $1675 \mathrm{~cm}^{-1}$. A second strong vibration peak, which increases with reaction time, was observed at $993 \mathrm{~cm}^{-1}$. The rate of formation of the peak at $993 \mathrm{~cm}^{-1}$ is faster than that of the peak at $1675 \mathrm{~cm}^{-1}$ (Figure 1), indicating that they belong to two different species. The change in the vibrations of the aromatic ring, of the methoxy groups and of the $\mathrm{C}-\mathrm{H}$ bonds in the aldehyde compared to the alcohol are seen as shifts in vibration frequencies. ${ }^{[29]}$

Interestingly, the vibration at $993 \mathrm{~cm}^{-1}$ only appears when both, Co(salen) and veratryl alcohol, are present. This is shown in Figure 2. A weak vibration peak at $984 \mathrm{~cm}^{-1}$ is observed to appear when only $\mathrm{Co}($ salen) is stirred at $\mathrm{pH} 12$ in the absence of oxygen, but when substrate is added, a strong vibration peak forms at $993 \mathrm{~cm}^{-1}$ and the vibration at $984 \mathrm{~cm}^{-1}$ can no longer be observed. Neither one of the peaks is observed at a lower $\mathrm{pH}$ (no addition of $\mathrm{NaOH}$, corresponds to a $\mathrm{pH}$ of about 9.5).
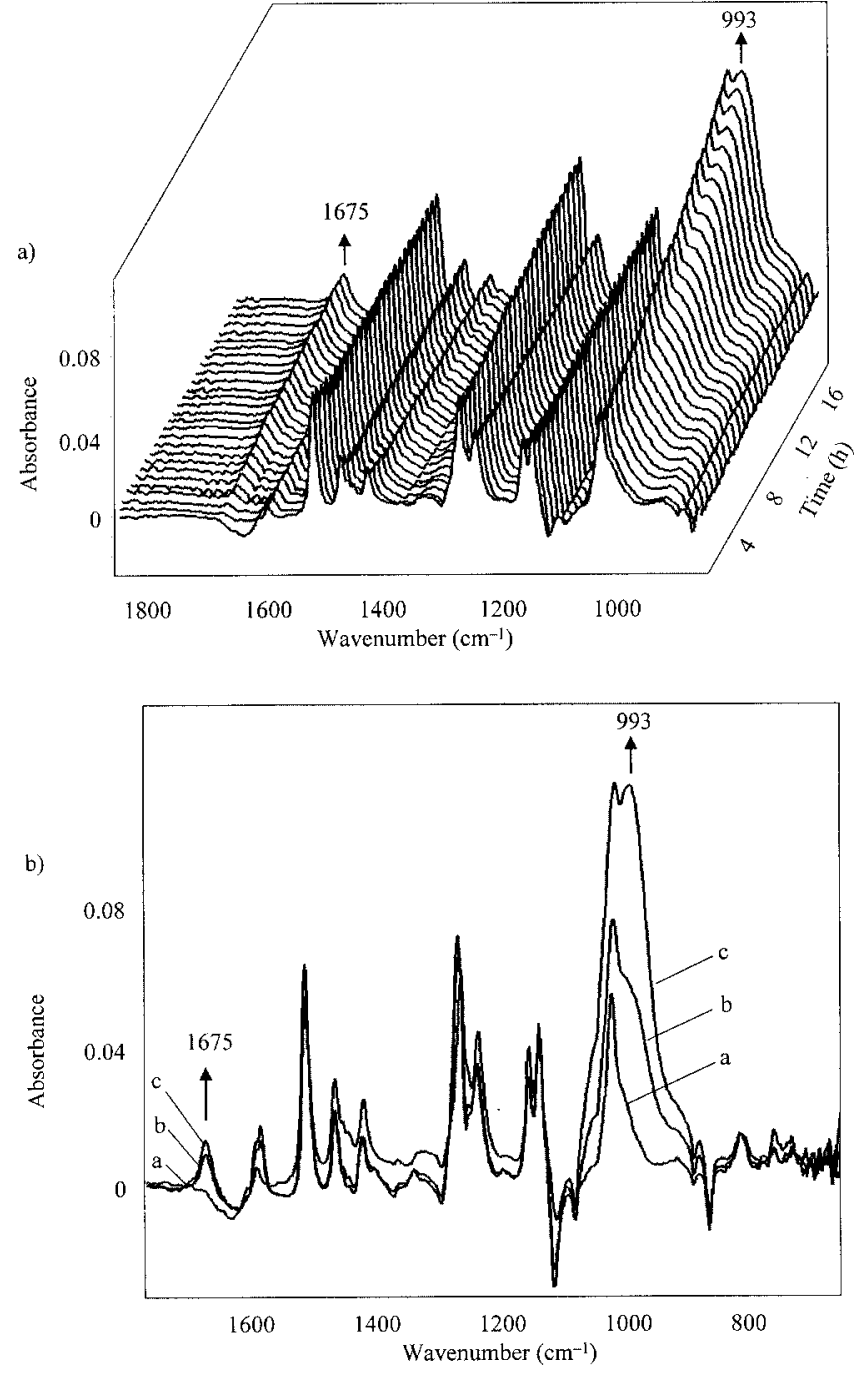

Figure 1. a) 3D plot of time-resolved ATR-IR spectra of the veratryl alcohol oxidation by $\mathrm{Co}\left(\right.$ salen) and dioxygen in water at $80^{\circ} \mathrm{C}$ and $\mathrm{pH}$ 12. b) Sample spectra were taken at $0 \mathrm{~h}$ (a), $8 \mathrm{~h} \mathrm{(b)} \mathrm{and} 16$ $\mathrm{h}$ (c). (150 mg Co(salen) and $3 \mathrm{~mL}$ of veratryl alcohol in $90 \mathrm{~mL}$ of water/dioxane (5:1) mixture).

Hydroxo-metal complexes exhibit the $\mathrm{MOH}$ bending mode below $1200 \mathrm{~cm}^{-1}$. If the hydroxo group is bridging two metal ions, the bending mode moves to lower energies and is seen at $955 \mathrm{~cm}^{-1}$ for bis- $\mu$-hydroxo[Cu(bipy $\left.)_{2}\right]$ type complexes. ${ }^{[24]}$ Thus, we assign the $984 \mathrm{~cm}^{-1}$ vibration to bis$\mu$-hydroxo[Co(salen) $]_{2}$ species as it appears in aqueous solution of $\mathrm{Co}($ salen) at $\mathrm{pH}$ 12. Metal-bound alkoxides (MOR) exhibit $v(\mathrm{C}-\mathrm{O})$ at ca. $1000 \mathrm{~cm}^{-1} \cdot{ }^{[24]}$ As the $993 \mathrm{~cm}^{-1}$ vibration appears in solutions of veratryl alcohol and Co(salen) at $\mathrm{pH} 12$ under argon, we assign this vibration to a cobalt-bound veratryl alkoxo group.

If this assignment is valid, the vibration at $993 \mathrm{~cm}^{-1}$ should behave differently in the absence or presence of oxygen. This is indeed the case, as illustrated in Figure 3. The rate of formation of the $993 \mathrm{~cm}^{-1}$ peak decreases after the addition of oxygen. This is also the point at which the production of aldehyde starts. The peak at $993 \mathrm{~cm}^{-1}$ does not 

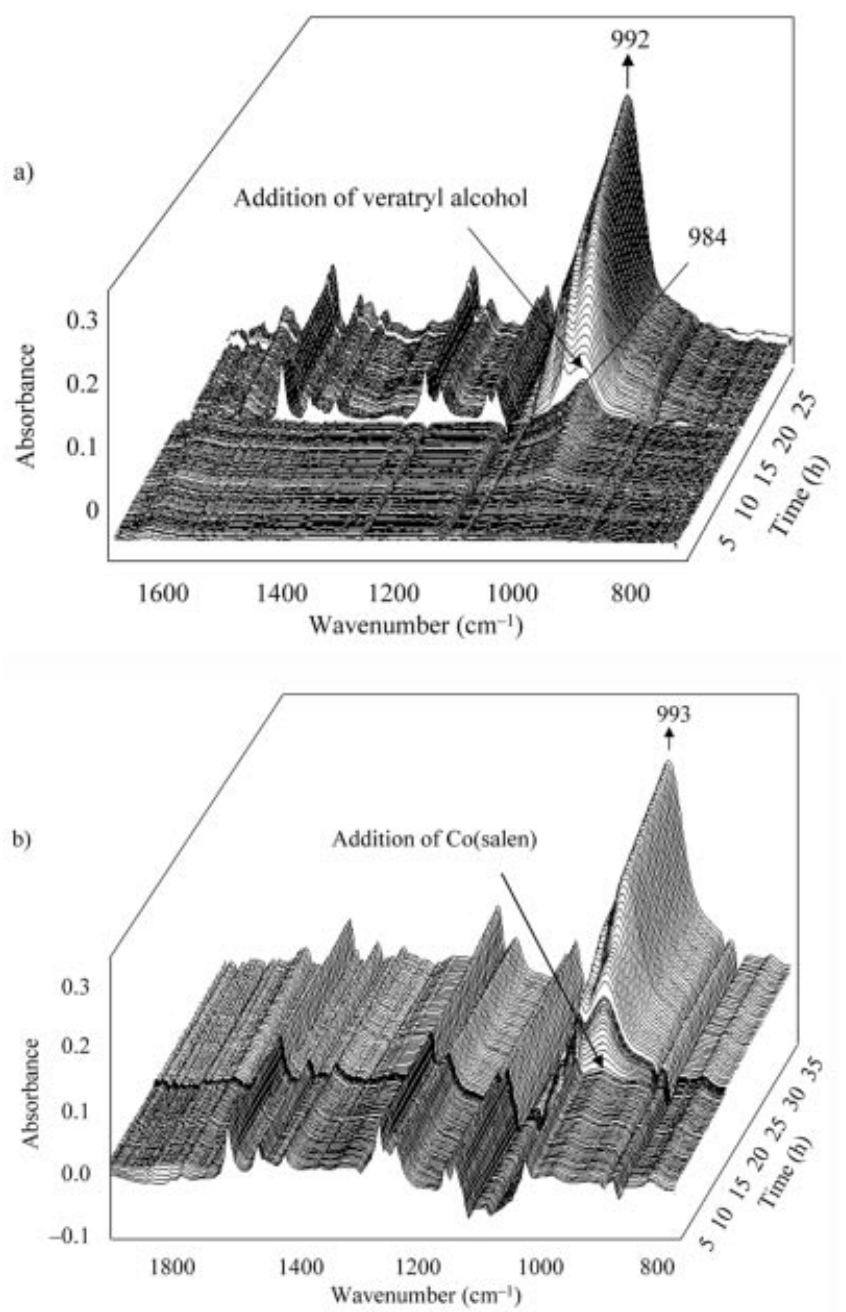

Figure 2. A strong vibration peak at $993 \mathrm{~cm}^{-1}$ appears after addition of veratryl alcohol (a) or Co(salen) (b) to the reaction mixture with other reagents. $[150 \mathrm{mg} \mathrm{Co}($ salen) and $3 \mathrm{~mL}$ of veratryl alcohol in $90 \mathrm{~mL}$ of water/dioxane $(5: 1)$ mixture at $80^{\circ} \mathrm{C}$ and $\mathrm{pH}$ 12 under argon].

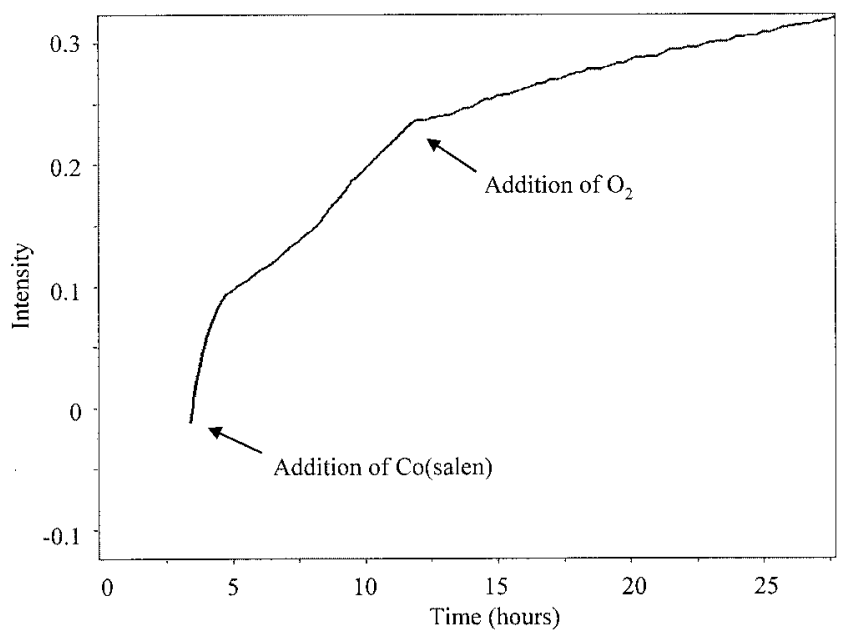

Figure 3. Appearance and increase in intensity of the vibration peak at $993 \mathrm{~cm}^{-1}$ monitored in a $\mathrm{Co}$ (salen) reaction in the absence and presence of oxygen. (150 mg Co(salen) and $3 \mathrm{~mL}$ of veratryl alcohol in $90 \mathrm{~mL}$ of water/dioxane $(5: 1)$ mixture at $80^{\circ} \mathrm{C}$ and $\mathrm{pH}$ 12 initially under argon). appear at temperatures lower than $70^{\circ} \mathrm{C}$ nor at low $\mathrm{pH}$ values (with no addition of $\mathrm{NaOH}$ ).

Summarizing, a vibration at $993 \mathrm{~cm}^{-1}$ appears at a temperature and $\mathrm{pH}$ range of an active system, but forms faster in the absence of oxygen. Thus it does not arise from the product nor from a oxygen-cobalt species, but from an interaction between the substrate and the complex. We assign this species to alkoxo-cobalt intermediate (Figure 3).

\section{Raman Spectroscopy}

Different oxygen bridges are more Raman or IR active depending on their symmetry and therefore it is important to use both techniques to determine the presence of oxygenbridged cobalt complexes. ${ }^{[19,24]}$ Raman spectroscopy is, however, even less sensitive than ATR-IR spectroscopy, and therefore a high concentration of reagents had to be used. The substrate exhibits vibrations at about the same frequencies as in the IR spectra. Figure 4 shows, that the only changes observed after an overnight measurement are the formation of a weak carbonyl vibration at $1675 \mathrm{~cm}^{-1}$ and shifts in the vibration peaks of the substrate. ${ }^{[29]}$ Thus no peroxo or superoxo bridges could be observed in the Raman spectra of the Co(salen)-catalyzed reaction.

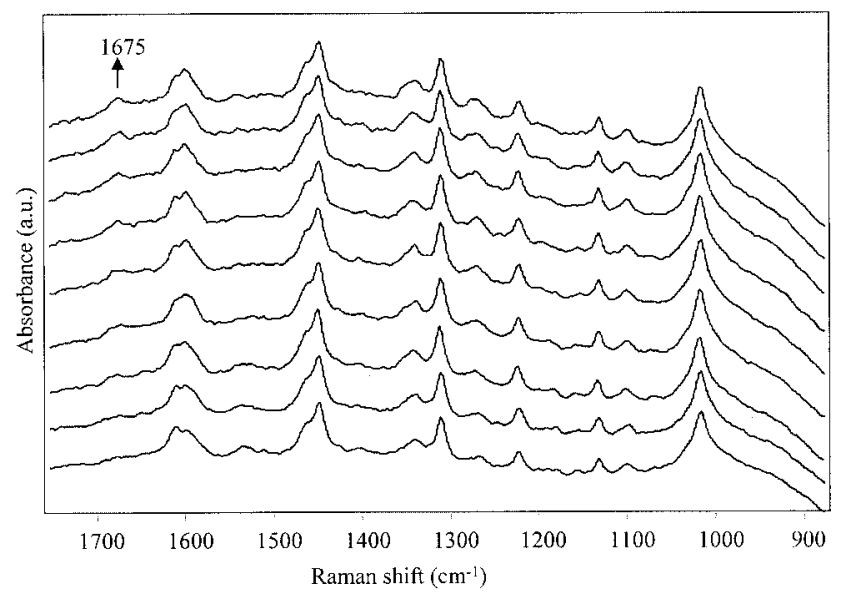

Figure 4. Raman spectra of Co(salen)-catalyzed veratryl alcohol oxidation. Vibration at $1675 \mathrm{~cm}^{-1}$ corresponds to the $\mathrm{C}=\mathrm{O}$ bond of the product. Other peaks observed belong to the substrate. ${ }^{[29]}$ (200 mg Co(salen) and $5 \mathrm{~mL}$ veratryl alcohol in $90 \mathrm{~mL}$ of water/ dioxane mixture $(3: 1)$ at $80{ }^{\circ} \mathrm{C}$ and $\left.\mathrm{pH} 12\right)$.

\section{UV/Vis Spectroscopy}

The oxygen complexes of cobalt have been widely studied by UV/Vis spectroscopy. ${ }^{[30-33]}$ According to Lever and Gray, the bridging superoxo complexes of cobalt exhibit a single intense band in the electronic spectra in the region $300-400 \mathrm{~nm}$, whereas a bridging peroxo has two absorption bands, at about $320 \mathrm{~nm}$ and $400 \mathrm{~nm}$. A doubly bridged $\mu$ hydroxo- $\mu$-peroxo $\left(\mathrm{OH}, \mathrm{O}_{2}{ }^{2-}\right)$ has only one intense absorption band at about $375 \mathrm{~nm}$ and a shoulder at higher energies (about $290 \mathrm{~nm}$ ). ${ }^{[30]}$ Aldehydes also exhibit absorptions in the UV range arising from the $\mathrm{n} \rightarrow \pi^{*}$ transitions of the 
free electron pair of the carbonyl oxygen and thus product formation can also be monitored by UV/Vis spectroscopy. ${ }^{[34]}$

Figure 5 shows the UV/Vis spectra of an experiment, in which $\mathrm{NaOH}$ was added to the reaction solution of Co(salen) and veratryl alcohol before oxygen (reactions 1-4). $\mathrm{Co}$ (salen) and veratryl alcohol in water at $80^{\circ} \mathrm{C}$ and under argon exhibit absorption bands of similar intensity at $339 \mathrm{~nm}$ and $392 \mathrm{~nm}$ (1). At pH 12 the intensity of the absorption bands is enhanced, but they do not shift (2). Addition of an oxygen atmosphere shifts the absorption bands to $324 \mathrm{~nm}$ and $378 \mathrm{~nm}$ (3). Under argon the reaction solution is very reddish brown, but it turns to dark brown along exposure to oxygen. Under prolonged oxygen exposure the intensity of the absorption band at $324 \mathrm{~nm}$ decreases to a shoulder, while the band at $378 \mathrm{~nm}$ remains (4). This change suggests that a monobridged peroxo species transforms into a doubly bridged species. At these conditions this is most likely a $\mu$-hydroxo- $\mu$-peroxo bridge. ${ }^{[35]}$

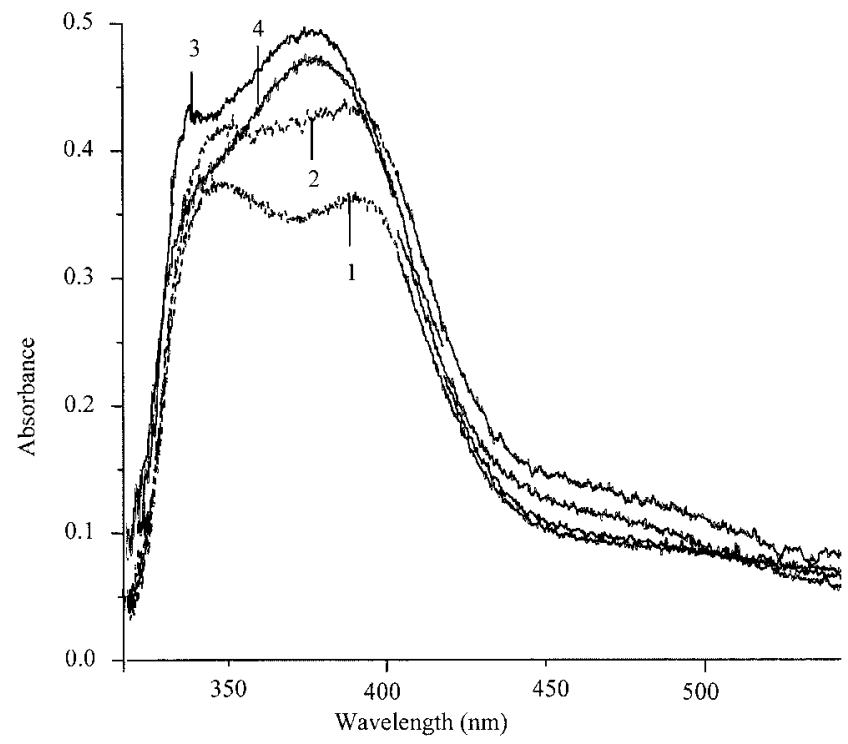

Figure 5. In-situ UV/Vis spectra of a solution of Co(salen) and veratryl alcohol in water under argon (1), after addition of $\mathrm{NaOH}$ to adjust $\mathrm{pH}$ to 12 (2), after addition of oxygen (3) and after $1 \mathrm{~h}$ of oxygen exposure (4). (10 $\mathrm{mg}$ of $\mathrm{Co}$ (salen) and $2 \mathrm{~mL}$ of veratryl alcohol in $120 \mathrm{~mL}$ of water at $80^{\circ} \mathrm{C}$ ).

If oxygen is added to a solution of $\mathrm{Co}($ salen) and veratryl alcohol at low $\mathrm{pH}$ (no addition of $\mathrm{NaOH}$ ), the absorption bands at $339 \mathrm{~nm}$ and $390 \mathrm{~nm}$ (5) are shifted to $300 \mathrm{~nm}$ and $378 \mathrm{~nm}$ (6). This implies that oxygen coordinates to the cobalt complex. After the addition of $\mathrm{NaOH}$, the band at $378 \mathrm{~nm}$ does not shift, but an absorption band at $308 \mathrm{~nm}$ starts to arise, which is assigned to the $\mathrm{n} \rightarrow \pi^{*}$ transitions in the $\mathrm{C}=\mathrm{O}$ group of the aldehyde (7, Figure 6$).{ }^{[34,36]}$

Aldehyde formation can be monitored by UV/Vis spectroscopy as the appearance and growth of the absorption band at $308 \mathrm{~nm}$ and Figure 7 shows that the reaction rate increases with increasing reaction temperature. At $40^{\circ} \mathrm{C}$ the rate of the reaction is so slow that practically no aldehyde is produced. Instead, at $60{ }^{\circ} \mathrm{C}$ the absorption band at

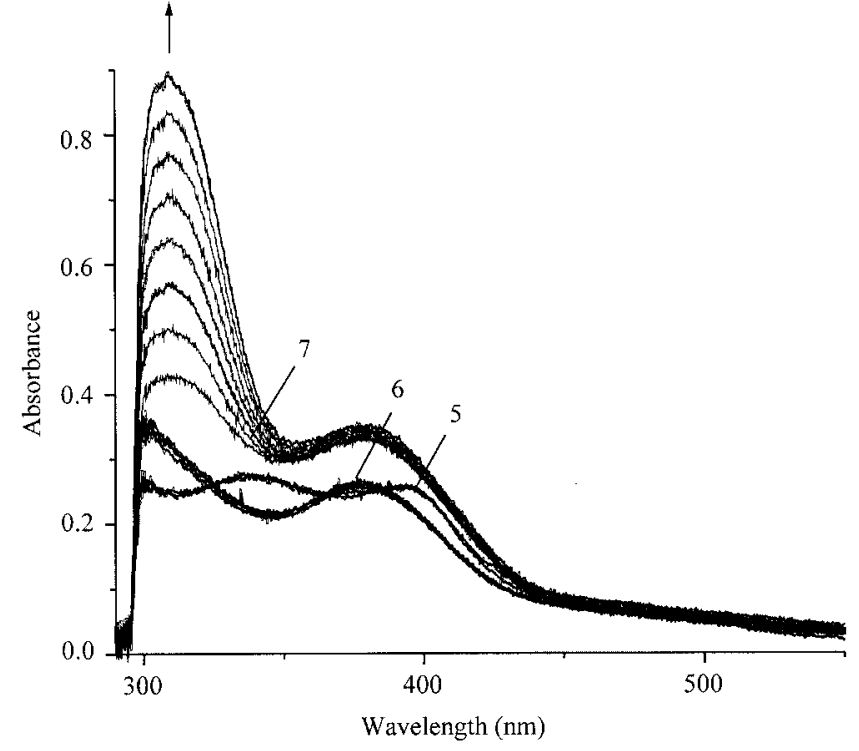

Figure 6. In-situ UV/Vis spectra of a solution of Co(salen) and veratryl alcohol in water at $80^{\circ} \mathrm{C}$ under argon (5), after addition of oxygen (6) and after addition of $\mathrm{NaOH}$ to adjust $\mathrm{pH}$ to 12 (7). (10 mg of Co(salen) and $2 \mathrm{~mL}$ of veratryl alcohol in $120 \mathrm{~mL}$ of water at $80^{\circ} \mathrm{C}$ ).

$308 \mathrm{~nm}$ starts to appear and at $80^{\circ} \mathrm{C}$ its formation rate increases dramatically.

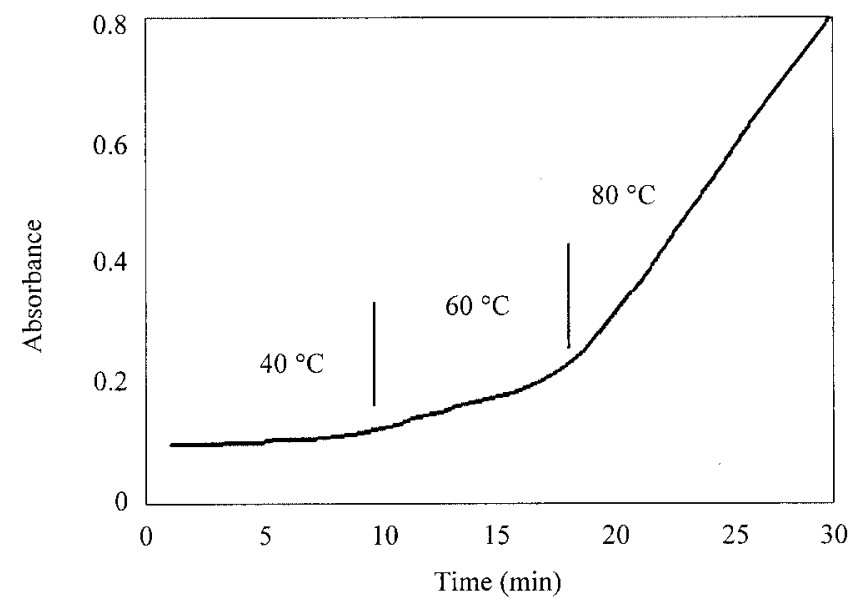

Figure 7. The rate of formation of the absorption band at $308 \mathrm{~nm}$ at different reaction temperatures.

Summarizing, a $\mu$-peroxo bridge is observed to form when oxygen is introduced to the solution of $\mathrm{Co}$ (salen) and veratryl alcohol in water at $80^{\circ} \mathrm{C}$ and $\mathrm{pH} 12$. With time, a second bridge appears between the cobalt centers forming a doubly bridged $\mu$-hydroxo- $\mu$-peroxo species. Coordination of veratryl alcohol to the cobalt center followed by insertion of oxygen can also be seen at lower $\mathrm{pH}$, but aldehyde formation does start before adjusting the $\mathrm{pH}$ to 12 . Increasing temperature considerably enhances the rate of aldehyde production.

\section{Reaction Monitoring Experiments}

In our earlier studies it has been shown that substrate concentration strongly affects the activity of the system. It 
was especially noted that the substrate to $\mathrm{Co}($ salen) ratio needs to be high for higher activity. ${ }^{[16]}$ The formation of high amounts of an intermediate, in which the substrate is coordinated to cobalt at high reagent concentrations, can be observed in the ATR-IR spectra. Addition of oxygen slows down the formation of this species and initiates the formation of the aldehyde. At high concentrations of all reagents a oxygen-cobalt species is not observed, whereas at the low concentrations of the UV/Vis experiments oxygen insertion is seen to occur. Thus, to study the influence of oxygen pressure on the reaction at high and low concentrations, reaction-monitoring experiments were performed with a set-up from which samples can be taken during the experiment under higher oxygen pressures. The initial rates of reaction were also calculated. ${ }^{[37]}$ Figure 8 summarizes the results of these experiments. At low concentrations [0.14 $\mathrm{mm} \mathrm{Co(salen)} \mathrm{and} 14 \mathrm{~mm}$ veratryl alcohol] the reaction rate is about the same under 1 and 10 bar oxygen pressure (Figure 8, experiments $\mathbf{1}$ and $\mathbf{2}$, initial rates of 9.5 and 9.6 $\mathrm{TOF}_{0}$, respectively ${ }^{[37]}$ ). At higher concentrations [0.41 mm Co(salen) and $41 \mathrm{~mm}$ veratryl alcohol] oxygen pressure significantly enhances the reaction rate (Figure 8 , experiments 3 and 4 , initial rates of 1.8 and $16 \mathrm{TOF}_{0}$, respectively).

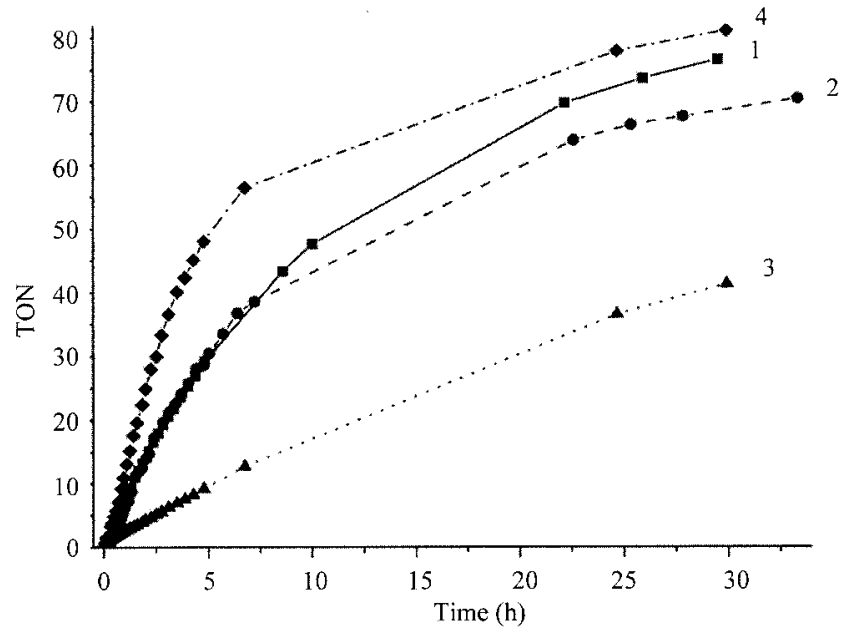

Figure 8. Oxidation activity (expressed as turnover number, TON, the moles of product per one mol of catalyst) at (1) $0.14 \mathrm{~mm} \mathrm{Co}$ (salen), $14 \mathrm{~mm}$ veratryl alcohol and 1 bar oxygen, (2) $0.14 \mathrm{~mm} \mathrm{Co-}$ (salen), $14 \mathrm{~mm}$ veratryl alcohol and 10 bar oxygen, (3) $0.41 \mathrm{~mm} \mathrm{Co-}$ (salen), $41 \mathrm{~mm}$ veratryl alcohol and 1 bar oxygen and (4) $0.41 \mathrm{~mm}$ $\mathrm{Co}($ salen), $41 \mathrm{~mm}$ veratryl alcohol and 10 bar oxygen.

Higher catalyst activity at low concentrations and low impact of added oxygen pressure on the catalytic system at low concentrations support the involvement of $2: 1 \mathrm{Co}: \mathrm{O}_{2}$ species in the catalytic cycle as the active species. In case of a mononuclear superoxo complex, increasing oxygen pressure should increase the reaction rate. ${ }^{[38]}$

As we have previously reported, the optimum $\mathrm{pH}$ for Co(salen)-catalyzed oxidation of veratryl alcohol is about 12.5. ${ }^{[16]}$ The initial oxidation rate of $\mathrm{Co}($ salen) increases from $5.2 \mathrm{~h}^{-1}$ to $9.6 \mathrm{~h}^{-1}$ when the $\mathrm{pH}$ is increased from 11.2 to 12.4 . If the activity and the change in $\mathrm{pH}$ of the reaction are monitored simultaneously (Figure 9), it can be seen that the product formation and the $\mathrm{OH}^{-}$ion consumption proceed hand in hand but no reasonable correlation between the conversions and the change in $\mathrm{pH}$ can be deduced. ${ }^{[39]}$ Figure 9 also shows that the decrease in $\mathrm{OH}^{-}$concentration slows down the reaction rate. Addition of $\mathrm{NaOH}$ at the end of a reaction restarts the oxidation activity. ${ }^{[16]}$

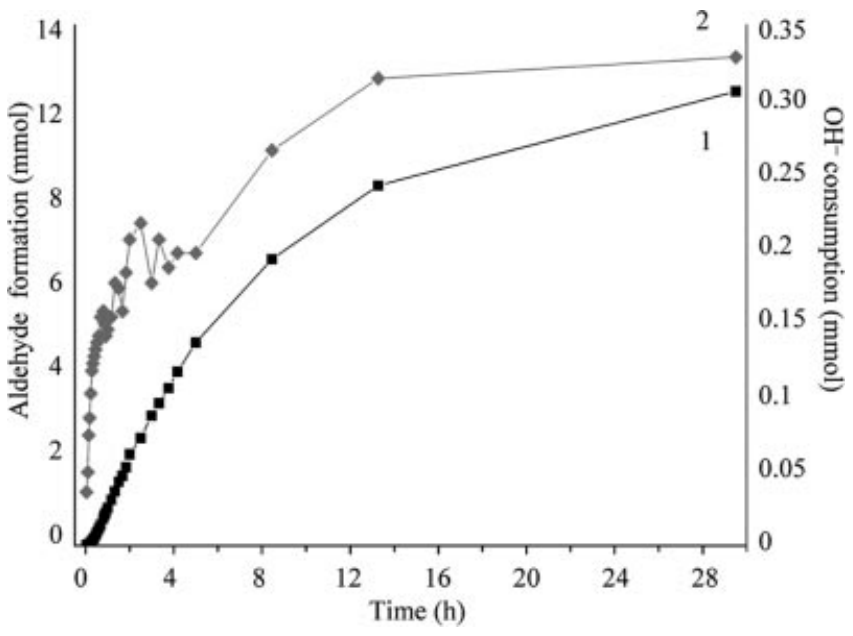

Figure 9. Rate of oxidation and total $\mathrm{OH}^{-}$consumption in the Co(salen)-catalyzed reaction: (1) Formation of veratraldehyde and (2) consumption of $\mathrm{OH}^{-}$ions. [0.14 mM Co(salen) and $14 \mathrm{~mm}$ veratryl alcohol at $\mathrm{pH} 11.6^{[40]}$ and $\left.80^{\circ} \mathrm{C}\right]$ (TON: turnover number).

\section{Discussion}

Based on the presented results and supported by our earlier studies, ${ }^{[16-17]}$ the oxidation of veratryl alcohol in water catalyzed by $\mathrm{Co}$ (salen) and oxygen is suggested to proceed through the steps described in Scheme 2.

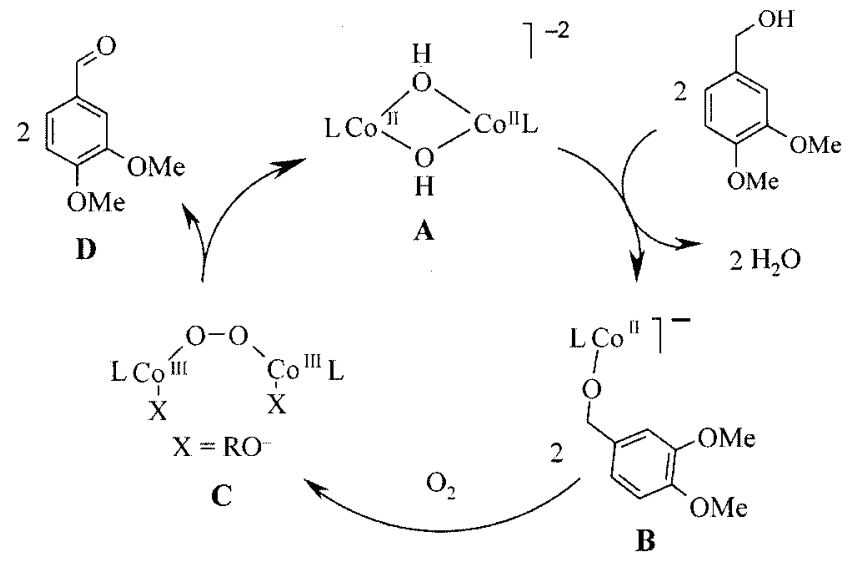

Scheme 2. Suggested mechanism for veratryl alcohol oxidation by $\mathrm{Co}($ salen) and dioxygen in alkaline aqueous solution.

The cycle of veratryl alcohol oxidation starts with a bis$\mu$-hydroxo[Co(salen) $]_{2}$ complex $\mathbf{A}$, which can deprotonate a substrate molecule at temperature higher than $60^{\circ} \mathrm{C}$ forming an alkoxo intermediate $\mathbf{B}$ and water. The intermediate B can be observed in the ATR-IR spectra. In the absence of oxygen, this species accumulates in the reaction. After 
introduction of oxygen, the formation of a nonplanar $\mu$ peroxo bridge between two alkoxy-Co(salen) units can be observed in UV/Vis spectra (intermediate $\mathbf{C}$ ). The peroxo bridge of species $\mathbf{C}$ abstracts a proton from each substrate and veratraldehyde is formed as a product (D).

In the overall reaction, one activated oxygen molecule oxidizes two veratryl alcohol molecules to veratraldehyde, water is produced as the side product and the initial bis- $\mu$ hydroxo $[\mathrm{Co}(\text { salen })]_{2}$ complex is regenerated in the cycle. The rate-limiting step seems to be the detachment of the product molecule. The methoxy substituents in the aromatic ring of veratryl alcohol seem to aid this step. ${ }^{[41]}$ Table 1 summarizes the spectroscopic fingerprints of species A-D. Below the proposed steps of the mechanistic cycle are discussed in more detail.

Table 1. Spectroscopic fingerprints of species A-D (sh = shoulder).

\begin{tabular}{llll}
\hline Species & ATR-IR $\left[\mathrm{cm}^{-1}\right]$ & $\operatorname{Raman}\left[\mathrm{cm}^{-1}\right]$ & UV/Vis $[\mathrm{nm}]$ \\
\hline A & 984 & - & $316,375,490 \mathrm{sh}$ \\
B & 993 & - & 339,392 \\
C & - & - & 324,378 \\
D & 1675 & 1677 & 308 \\
\hline
\end{tabular}

\section{Formation of Complex A}

The reaction does not start before the addition of $\mathrm{NaOH}$, and therefore it is apparent that $\mathrm{OH}^{-}$ions are directly involved in generating the active species and not only acting as stoichiometric proton acceptors. As the $\mathrm{pH}$ gets lower during the reaction, possibly due to the formation of small amounts of veratrylic acid, ${ }^{[16]}$ the concentration of the active species decreases and consequently the reaction rate slows down.

The catalytic activity can be regained by adding $\mathrm{NaOH}$ to the solution, ${ }^{[16]}$ which is a clear indication that the active catalytic species is present only at higher $\mathrm{pH}$. It has been shown that at higher $\mathrm{pH}$ values the $\mathrm{Co}($ salen) complex exists rather as a hydroxo complex. ${ }^{[35,42]}$ As the $\mathrm{pH}$ decreases the concentration of the hydroxo complex also decreases and the reaction slows down, whereas addition of $\mathrm{NaOH}$ shifts the equilibrium back towards the hydroxo species and the rate of reaction increases again. At the applied conditions in the beginning of the reaction, it is reasonable to expect, that the Co(salen) hydroxo complex exists most likely as a dimeric bis- $\mu$-hydroxo[Co(salen) $]_{2}$ species A. ${ }^{[35,38,43]}$ This species exhibits a vibration at $984 \mathrm{~cm}^{-1}$ (arising from bridging $\mathrm{OH}$ groups) and absorption bands at $316 \mathrm{~nm}$ and $375 \mathrm{~nm}$ with a shoulder at $490 \mathrm{~nm}$.

\section{Formation of the Intermediate B}

According to UV/Vis spectroscopy, the substrate can replace the hydroxy group from the first coordination sphere of the cobalt center. This is seen as a shift of the absorption bands at $316 \mathrm{~nm}$ and $375 \mathrm{~nm}$ to $336 \mathrm{~nm}$ and $392 \mathrm{~nm}$ after addition of veratryl alcohol to the solution of $\mathrm{Co}($ salen) at $\mathrm{pH}$ 12. It was already suggested before, that substrate coor- dination must be necessary for the reaction to occur as the strongly binding axial bases inhibit the reaction and the high substrate to metal ratio enhances it. ${ }^{[16-17]}$ ATR-IR spectra show clearly that an intermediate species is formed between veratryl alcohol and $\mathrm{Co}($ salen). This intermediate B exhibits a strong vibration at $993 \mathrm{~cm}^{-1}$ corresponding to the $\mathrm{C}-\mathrm{O}$ bond of the metal-bound alkoxo group. ${ }^{[24]}$ The vibration at $993 \mathrm{~cm}^{-1}$, however, is observed to accumulate, especially in the absence of oxygen. The rate at which the intermediate $\mathbf{B}$ forms decreases after introduction of oxygen, as from that point on the aldehyde production starts. Nevertheless, the vibration peak at $993 \mathrm{~cm}^{-1}$ still grows. In other words, formation of species $\mathbf{B}$ is a fast step compared to the formation of species $\mathbf{C}$ and $\mathbf{D}$.

The need of higher reaction temperatures is due to the formation of the alkoxo-metal intermediate $\mathbf{B}$. The formation of the strong vibration peak at $993 \mathrm{~cm}^{-1}$ is not observed at $60{ }^{\circ} \mathrm{C}$, but at $80^{\circ} \mathrm{C}$ it accumulates. Also, a high $\mathrm{pH}$ is needed for the vibration $993 \mathrm{~cm}^{-1}$ to appear. Coordination of the substrate as an alcohol to cobaltcenter occurs also at low $\mathrm{pH}$, as seen in UV/Vis spectra, but formation of the alkoxo species $\mathbf{B}$ requires deprotonation of the substrate. This seems to take place only at high temperature and $\mathrm{pH}$, as seen by the appearance of the $993 \mathrm{~cm}^{-1}$ peak. $\mathrm{UV} / \mathrm{Vis}$ spectroscopy shows as well that no aldehyde is formed at temperature below $60^{\circ} \mathrm{C}$ and that raising temperature to $80^{\circ} \mathrm{C}$ dramatically increases the rate of the reaction. Thus, the rate of reaction is dependent on the rate at which the substrate deprotonates and forms the intermediate $\mathbf{B}$.

\section{Formation of the Intermediate C}

As discussed above, the introduction of oxygen atmosphere is needed to start the production of aldehyde. ${ }^{[34]} \mathrm{UV} /$ Vis spectra show shifts in the absorption bands of cobalt after addition of oxygen to a solution formerly under argon. These shifts suggest a formation of a peroxo bridge between two Co(salen) complexes. ${ }^{[30-32]}$ Absorptions at $300 \mathrm{~nm}$ and $380 \mathrm{~nm}$ are observed when oxygen is added to a solution of $\mathrm{Co}($ salen) and veratryl alcohol at low $\mathrm{pH}$ (below 10). Aldehyde production does not start in this solution before addition of $\mathrm{NaOH}$. When oxygen is added to a solution of $\mathrm{Co}($ salen) and veratryl alcohol at $\mathrm{pH} 12$, absorption bands form at $324 \mathrm{~nm}$ and $392 \mathrm{~nm}$. These absorptions are defined as arising from monobridged $\mu$-peroxo complexes. ${ }^{[30,33]}$ Thus, it is apparent that an oxygen bridge forms between two $\mathrm{Co}$ (salen) complexes under these reaction conditions and that the formation of the intermediate $\mathbf{C}$ is needed for the aldehyde production to start. A similar species has been reported in the oxidation of benzyl alcohol by $\mathrm{Co}^{\mathrm{II}}(\text { bipy })_{2}$ complexes. ${ }^{[4]]}$

The charge and geometry of the oxygen-bridged complex should be observable from the ATR-IR and Raman spectra. Superoxo and peroxo bridges exhibit vibrations at frequency ranges of $1100-1200 \mathrm{~cm}^{-1}$ and $760-920 \mathrm{~cm}^{-1}$, respectively. ${ }^{[24]}$ Nevertheless, no vibration peaks are ob- 
served to form in these regions in neither ATR-IR nor Raman spectra. The reason arises from the effect of concentration on the activity of the system. At the high concentrations needed for the ATR-IR and Raman measurements, the oxidation activity is quite low without an added oxygen pressure, as seen in Figure 8. The intermediate $\mathbf{B}$ forms in high amounts in these conditions and it could be regarded as a "dormant" species, whenever there is not enough oxygen to take the reaction further. Therefore, at the conditions of ATR-IR and Raman measurements the concentration of the oxygen-bridged species $\mathbf{C}$ is too low to be observed. However, the UV/Vis spectra are recorded at low concentrations and in these spectra the oxygen-bridged species $\mathbf{C}$ can easily be observed.

According to the literature, the peroxo bridge most probably forms through a mononuclear superoxo complex. ${ }^{[8,12]}$ Oxygen molecule binds first to one cobalt center as a superoxo radical, which then binds to another cobalt complex forming a peroxo species. At these reaction conditions the dinuclear species is more stable and it forms fast. Thus the superoxo complex is so short-lived that it cannot be observed with the applied spectroscopic methods.

\section{The Rate-Limiting Step}

Alkoxo-metal species as a reaction intermediate have also been suggested for Co complexes mimicking horse liver alcohol dehydrogenase. In these reactions the product dissociation from the metal center was observed to be the rate-limiting step. ${ }^{[45]}$ This is apparently also the case for the reaction under study. We have observed that a alkoxo-metal species similar to intermediate $\mathbf{B}$ is also formed in the reaction of Co(salen) with benzyl alcohol, although benzaldehyde is not produced by this system. ${ }^{[41]}$ Thus, the reaction with benzyl alcohol seems to stop at the intermediate B, while with veratryl alcohol the aldehyde production starts directly after oxygen is introduced to the system. Thus, the methoxy substituents in veratryl alcohol aid the detachment of the product from the Co(salen) complex. Moreover, no alkoxo-metal intermediate is observed in the ATR-IR spectra of a similar system, in which (phenanthroline)coppertype $[\mathrm{Cu}($ phen) $)]$ complexes are used as catalysts. ${ }^{[46]}$ These complexes are more active than $\mathrm{Co}($ salen) and can also catalyze the oxidation of benzyl alcohol to some extent. This implies that the alkoxo-metal intermediate is less stable or that such species does not even form in the $\mathrm{Cu}$ (phen)-catalyzed system. ${ }^{[23,46]}$ The detachment step is preceded by abstraction of a hydrogen from the coordinated alkoxyl by the peroxo bridge. Thus, the methoxy substituents can be involved in making the $\alpha-\mathrm{H}$ in the benzylic position more labile and easier to abstract.

\section{The Regeneration of Complex A}

High $\mathrm{pH}$ is needed to generate the bis- $\mu$-hydroxo[Co(salen)] $]_{2}$ species $\mathbf{A}$, which can deprotonate the substrate. It seems that the overall $\mathrm{OH}^{-}$consumption and alcohol oxi- dation are closely related, but the molar ratio of formed aldehyde and $\mathrm{OH}^{-}$ions consumed varies from tens to hundreds. ${ }^{[16,39]}$ This indicates that actually only a few substrate molecules are deprotonated by $\mathrm{OH}^{-}$ions or that the $\mathrm{OH}^{-}$ ions are recovered during the reaction cycle. The latter suggestion supports the idea, that peroxo-bridged species $\mathbf{C}$ regenerates the bis- $\mu$-hydroxo[Co(salen) $]_{2}$ species by abstracting a hydrogen from each of the two coordinated substrate molecules. Therefore, after initiation no "external" hydroxide ions are consumed in the reaction. Nevertheless, if the $\mathrm{pH}$ gets too low due to small amount veratryl acid formed as a side-product, the cobalt complex prefers to stay as a peroxo-bridged species $\mathbf{C}$ and the reaction stops. Thus addition of $\mathrm{NaOH}$ increases the reaction rate as it enhances the deprotonation step and keeps the species $\mathbf{A}$ as the dominating species in the solution.

A drawback of the Co(salen) catalytic system is that it suffers from irreversible oxidation. We have observed that this system can stay active for about 72-96 h, after which there is no more oxidation occurring even with further addition of $\mathrm{NaOH}$. Thus, there is all the time a competing mechanistic path present, which causes the catalyst to deactivate. This could occur via the formation of doubly bridged $\mu$-hydroxo- $\mu$-peroxo species, which can be very stable. ${ }^{[35]}$ $\mathrm{UV} / \mathrm{Vis}$ spectra show a transformation in the bridging type of the oxygen complex from singly bridged peroxo towards a doubly bridged species. The presence of the substrate seems to protect the complex from deactivation. The activity is higher if the substrate to $\mathrm{Co}$ (salen) ratio is kept high. ${ }^{[16]}$ Possibly, the coordinated substrate can prevent the insertion of a second bridge between cobalt centers. Careful balancing of the reaction conditions can minimize the damage caused by deactivation. For example, keeping the Co(salen) concentration low and veratryl alcohol concentration high dramatically improves the lifetime of the catalyst. ${ }^{[16]}$

\section{Conclusions}

The activities of transition-metal complex-catalyzed alcohol oxidations have remained low, normally in the order of TOF $=10 \mathrm{~h}^{-1}$ or less, ${ }^{[4]}$ and therefore it is imperative to study the mechanisms of these reactions to be able to develop more active systems. In this work, the mechanism of $\mathrm{Co}$ (salen)-catalyzed oxidation of veratryl alcohol in alkaline aqueous solution was investigated in detail by in-situ spectroscopy. It was shown, that the combination of complementary in-situ spectroscopic techniques is a powerful approach to study a complicated catalytic system.

The obtained data support strongly a mechanism, in which substrate coordination is a key step in the catalytic cycle. Deprotonation and coordination of the substrate have been suggested before as reaction steps in similar systems, but direct proof is hard to find. Combination of in-situ UV/ Vis and ATR-IR spectroscopy show, that veratryl alcohol is located in the coordination sphere of cobalt already at low $\mathrm{pH}$ values, but deprotonation and consequent alkoxo- 
cobalt intermediate formation occurs only at $\mathrm{pH} 12$ and above. The spectroscopic data also suggests that a dinuclear $\mu$-peroxo $[\mathrm{Co}(\text { salen })]_{2}$ complex is involved in this oxidation reaction instead of the mononuclear superoxo-Co(salen) complex, which is usually assumed to be responsible for catalytic activity. The mononuclear superoxo complex can still be formed initially, but its lifetime is too short to be observed. Nevertheless, the effect of reaction conditions presented in earlier papers point strongly to the dinuclear peroxo species over the mononuclear superoxo complex as the active intermediate in the catalytic cycle of this particular reaction. ${ }^{[16-17]}$ Thus, both the optimum reaction conditions and the spectroscopic data support the mechanism described in Scheme 2. Further studies with different benzylic alcohols can give more insight into the effect of substituents on the rate-limiting step. Also, additional spectroscopic methods, such as EXAFS, could give further evidence for the involvement of the dinuclear species in this reaction.

\section{Experimental Section}

Co(salen) (1) was purchased from Aldrich and used as received without further purification. 3,4-Dimethoxybenzyl alcohol (veratryl alcohol) was purchased from ACROS and Aldrich in 97\% purity. The $3 \%$ impurity is veratraldehyde, which has been taken into account when calculating conversions and turnover numbers. 1,4Dioxane, 3,4-dimethoxybenzaldehyde and 3,4-dimethoxybenzylic acid were purchased from Aldrich and used as received. The oxygen gas was obtained from AGA in Finland and from Hoek-Loos in The Netherlands and had a purity of $99 \%$. Online ATR-IR spectra were recorded with a Mettler Toledo ReactIR ${ }^{\mathrm{TM}} 1000$ spectrometer with a DiComp ${ }^{\mathrm{TM}}$ probe, which was fitted in a $100-\mathrm{mL}$ two-neck round-bottomed flask containing the reaction solution. The measurements were done with a scan program of 128 scans per measurement at selected intervals with a resolution of $4 \mathrm{~cm}^{-1}$. The wavelength range covered was $650-4000 \mathrm{~cm}^{-1}$. The diamond absorption prevents to observe any vibrations at a wavelength range of 1900-2400 $\mathrm{cm}^{-1}$. A more detailed description of the ATR-IR setup and the procedure of the oxidation experiments is presented elsewhere. ${ }^{[16-17,21]}$ The solvent used in all ATR-IR experiments was a water/dioxane mixture $(5: 1)$ at $80^{\circ} \mathrm{C}$, which was in all cases subtracted as the background. The amounts of reagents varied, and therefore their exact concentrations are specified at the appropriate sections of this paper or in relevant Figure captions. Oxygen was installed by the balloon technique. ${ }^{[16]}$ All reagents were degassed and under argon when working in the absence of oxygen. The IR spectra were analyzed with the program ReactIR 2.2. The UV/Vis spectra were recorded with a Varian CARY50 spectrometer equipped with an optical fibre and an insertion probe with variable path lengths. The same procedure for the oxidation experiments was used as with the ATR-IR technique. The Raman spectra were recorded with a Kaiser RXN dispersive system with a Peltier element-cooled Andor CCD camera for detection and a laser of $532 \mathrm{~nm}(60 \mathrm{~mW})$. In these experiments, the reaction was monitored from the outside of the glass flask by using a laser objective. The resolution was about $2 \mathrm{~cm}^{-1}$ and 10 scans were accumulated with an exposure time of $15 \mathrm{~s}$. The reaction monitoring experiments were performed in a $500-\mathrm{mL}$ glass vessel equipped with a magnetic stir-bar fitted inside a 1-L steel autoclave. $200 \mathrm{~mL}$ of $0.05 \mathrm{M} \mathrm{NaOH}$ $(\mathrm{pH}, 12.4)$ was used as solvent. Depending on the experiment, $86.9 \mathrm{mg}(0.275 \mathrm{mmol})$ or $269 \mathrm{mg}(0.83 \mathrm{mmol})$ of $\mathrm{Co}$ (salen $)$ was used. Veratryl alcohol was added in 100:1 molar ratio to Co(salen). The autoclave was placed in an oil bath and the reaction mixture was stirred at $80^{\circ} \mathrm{C}$ under 10 bar of oxygen pressure. Samples were cooled to room temperature and prepared for GC analysis by a method described earlier. ${ }^{[23]} \mathrm{GC}$ measurements were conducted with a GC/Fid Agilent $6890 \mathrm{~N}$ instrument using a HP-5 (30 m $\times$ $320 \mu \mathrm{m} \times 0.25 \mu \mathrm{m}$ ) capillary column and equipped with a FID detector.

\section{Acknowledgments}

Financial support from University of Helsinki, Academy of Finland (grant 204408, T.R.), Finnish Cultural Foundation (K. K.), Magnus Ehnrooth Foundation (K. K.), Acta Chemica Scandinavica (K. K.) and Finnish Technology Development Agency TEKES (H. K.) is gratefully acknowledged. B.M. W. and J.G.M. acknowledge funding from CW-NWO (VICI and Van der Leeuw grants) and NRSC-C. The authors also thank Dr. Tom Visser and Dr. Markus Allmendinger for valuable discussions.

[1] J. Ebner, D. Riley, in: Active Oxygen in Chemistry, (Eds.: C. S. Foote, J. S. Valentine, A. Greenberg, J. F. Liebman); SEARCH series, vol. 1, Chapman \& Hall, 1995, pp. 204-248.

[2] R. A. Sheldon, I. W. C. E. Arends, G.-J. ten Brink, A. Dijksman, Acc. Chem. Res. 2002, 35, 774-781.

[3] T. Elder, J. J. Bozell, Holzforschung 1996, 50, 24-30.

[4] Advances in Catalytic Activation of Dioxygen by Metal Complexes (Ed.: L. I. Simandi), Kluwer, Dordrecht, 2003.

[5] A. Lubineau, J. Augé, in: Aqueous-Phase Organometallic Catalysis (Eds.: B. Cornils, W. A. Herrmann), Wiley-VCH, Weinheim, 1998.

[6] A. Dijksman, A. Marino-González, A. Mairata i Payeras, I. W. C. E. Arends, R. A. Sheldon, J. Am. Chem. Soc. 2001, $123,6826-6833$

[7] B. A. Steinhoff, S. R. Fix, S. S. Stahl, J. Am. Chem. Soc. 2002, 124, 766-767.

[8] R. Sheldon, J. Kochi, Metal-Catalyzed Oxidations of Organic Compounds, Academic Press, 1981.

[9] A. Martell, in: Oxygen Complexes and Oxygen Activation by Transition Metals, (Eds.: A. Martell, D. Sawyer), Plenum Press, 1987.

[10] T. Tsumaki, Bull. Chem. Soc. Jpn 1938, 13, 252-257.

[11] D. Chen, A. E. Martell, Inorg. Chem. 1987, 26, 1026-1030.

[12] J. Bozell, B. R. Hames, D. R. Dimmel, J. Org. Chem. 1995, 60, 2398-2404.

[13] D. E. Hamilton, R. S. Drago, A. Zombeck, J. Am. Chem. Soc. 1987, 109, 374-379.

[14] A. Zombeck, R. S. Drago, B. B. Corden, J. H. Gaul, J. Am. Chem. Soc. 1981, 103, 7580-7585.

[15] M. M. Whittaker, J. W. Whittaker, Biochemistry 2001, 40, $7140-7148$.

[16] K. Kervinen, H. Korpi, M. Leskelä, T. Repo, J. Mol. Catal. A: Chemical 2003, 203, 9-19.

[17] K. Kervinen, P. Lahtinen, T. Repo, M. Svahn, M. Leskelä, Catal. Today 2002, 75, 183-188.

[18] Y. Cui, R. Patt, R. Chen, J. Gratzl, J. Mol. Catal. A: Chemical 1999, 144, 411.

[19] Introduction to Infrared and Raman Spectroscopy (Eds.: N. B. Colthup, L. H. Daly, S. E. Wiberley), Academic Press, Inc., San Diego, 1990.

[20] B. M. Weckhuysen, Phys. Chem. Chem. Phys. 2003, 5, 43514360.

[21] In-situ Spectroscopy of Catalysts (Ed.: B. M. Weckhuysen), American Scientific Publishers, Stevenson Ranch, 2004.

[22] K. Kervinen, M. Allmendinger, M. Leskelä, T. Repo, T. B. Rieger, Phys. Chem. Chem. Phys. 2003, 5, 4450-4454. 
[23] H. Korpi, P. Lahtinen, V. Sippola, O. Krause, M. Leskelä, T. Repo, Appl. Catal. A General 2004, 268, 199-206.

[24] Infrared and Raman Spectra of Inorganic and Coordination Compounds, Part B: Applications in Coordination, Organometallic and Bioinorganic Chemistry (Ed.: K. Nakamoto), John Wiley \& Sons, Inc., New York, 1997.

[25] N. N. Greenwood, A. Earnshaw, Chemistry of the Elements, Pergamon Press, Oxford, 1984.

[26] E. M. Nour, R. E. Hester, J. Mol. Struct. 1980, 62, 77-79.

[27] M. Suzuki, T. Ishiguro, M. Kozuka, K. Nakamoto, Inorg. Chem. 1981, 20, 1993-1996.

[28] C. G. Barraclough, G. A. Lawrence, P. A. Lay, Inorg. Chem. 1978, 17, 3317-3322.

[29] The reference spectra measured at $\mathrm{pH} 12.5$ in water/dioxane mixture $(75 \mathrm{~mL}: 15 \mathrm{~mL})$ show the following vibrations for a $0.4 \mathrm{M}$ solution of veratryl alcohol $\left(\mathrm{cm}^{-1}\right)$ : $2948(\mathrm{w}), 2923(\mathrm{w})$, $2892(\mathrm{w}), 2848$ (w), $1596(\mathrm{w}), 1517$ (s), $1468(\mathrm{~m}), 1445(\mathrm{w}), 1424$ (m), 1335 (m), 1266 (s), 1239 (m), 1158 (m-s), 1140 (m-s), 1025 (s), 816 (w). Veratraldehyde exhibits vibrations at $\left(\mathrm{cm}^{-1}\right): 2960$ (w), $2854(\mathrm{w}), 1729(\mathrm{w}), 1673(\mathrm{~m}), 1588$ (d, m), 1513 (m-s), $1468(\mathrm{~m}), 1455(\mathrm{w}), 1428(\mathrm{w}), 1345(\mathrm{w}), 1272(\mathrm{~s}), 1245(\mathrm{w}), 1160$ $(\mathrm{w}), 1140(\mathrm{~m}), 1071(\mathrm{w}), 1017(\mathrm{~m})$. Similar shifts are observed in the Raman spectra, but the intensities of the vibrations can differ.

[30] A. B. P. Lever, H. B. Gray, Acc. Chem. Res. 1978, 11, 348-355.

[31] Inorganic Electronic Spectroscopy (Ed.: A. B. P. Lever), Elsevier, Amsterdam, 1986.

[32] G. McLendon, R. J. Motekaitis, A. E. Martell, Inorg. Chem. 1975, 14, 1993-1996.

[33] V. M. Miskowski, J. L. Robbins, I. M. Treitel, H. B. Gray, Inorg. Chem. 1975, 14, 2318-2321.

[34] Spectroscopic Methods in Organic Chemistry (Eds.: D. H. Williams, I. Fleming); McGraw-Hill Book Company, London, 1989.

[35] R. D. Jones, D. S. Summerville, F. Basolo, Chem. Rev. 1979, 79, $139-179$.

[36] The commercial veratraldehyde exhibits absorption maxima at $268 \mathrm{~nm}$ and $308 \mathrm{~nm}$ with a shoulder at $290 \mathrm{~nm}$. Veratrylic acid shows absorption maximum at $250.4 \mathrm{~nm}$ with shoulders at $295 \mathrm{~nm}$ and $330 \mathrm{~nm}$.
[37] The initial oxidation rates were calculated from the derivatives at time zero for the functions presenting the conversion of the aldehyde as a function of time. These functions were obtained from the linear fit for the conversion points as a function of time during the first $10 \%$ of aldehyde formation. The rate of the reaction decreases after the conversion of aldehyde is over $15 \%$. The slope of the plot was multiplied with the substrate/ metal molar ratio to get result in the form of $\mathrm{TON} / \mathrm{h}=\mathrm{TOF}$ $(1 / h)$.

[38] E. C. Niederhoffer, J. H. Timmons, A. E. Martell, Chem. Rev. 1984, 84, 137-203.

[39] If the reaction is monitored according to the consumption of hydroxide ions vs. amount of aldehyde produced, more than ten equivalents of aldehyde is formed per consumed $\mathrm{OH}^{-}$ion. For example, in an overall reaction in which $\mathrm{pH}$ decreased from 11.2 to $10.0,30$ moles of aldehyde was produced per $1 \mathrm{~mol}$ of hydroxide consumed.

[40] In these reactions initial $\mathrm{pH} 11.6(0.005 \mathrm{~m} \mathrm{NaOH})$ was applied, as even modern $\mathrm{pH}$ electrodes seem to be too inaccurate for such a study in $\mathrm{pH}$ higher than 12 .

[41] Although unsubstituted benzyl alcohol is not oxidized by this catalytic system, the vibration peak at $993 \mathrm{~cm}^{-1}$ is also visible in the corresponding ATR-IR spectra.

[42] R. J. Motekaitis, A. E. Martell, Inorg. Chem. 1988, 27, 2718 2724.

[43] Comprehensive Coordination Chemistry: the synthesis, reactions, properties and applications of coordination compounds (Ed.: G. Wilkinson); vol. 4, Pergamon Press, 1987.

[44] A. Sobkowiac, D. T. Sawyer, J. Am. Chem. Soc. 1991, 113, 9520-9523.

[45] M. Gerber, M. Zeppezauer, M. F. Dunn, Inorg. Chim. Acta 1983, 79, 161-164.

[46] H. Korpi, K. Kervinen, T. Repo, M. Leskelä, J. Chem. Soc., Dalton Trans, submitted to.

[47] R. A. Sheldon, I. W. C. E. Arends, A. Dijksman, Catal. Today 2000, 57, 157-166.

Received: January 13, 2005 Published Online: April 25, 2005 